\title{
A Canonical Space-Time Characterization of Mobile Wireless Channels
}

\author{
Akbar M. Sayeed, Member, IEEE, Eko N. Onggosanusi, Student Member, IEEE, \\ and Barry D. Van Veen, Senior Member, IEEE
}

\begin{abstract}
A canonical space-time characterization of mobile wireless channels is introduced in terms of a fixed basis that is independent of the true channel parameters. The basis captures the essential degrees of freedom in the received signal using discrete multipath delays, Doppler shifts, and directions of arrival. This provides a robust representation of the propagation dynamics and dramatically reduces the number of channel parameters to be estimated. The resulting canonical space-time receivers deliver optimal performance at substantially reduced complexity compared to existing designs.
\end{abstract}

Index Terms - Dimension reduction, dispersive channels, space-time sampling.

\section{INTRODUCTION}

$\mathbf{T}$ THE USE OF antenna arrays for enhancing the capacity and quality of multiuser wireless communication systems has spurred significant interest in space-time signal processing techniques [1]. A key consideration in space-time receiver design is modeling the complex time-varying multipath propagation environment. Most existing receiver designs employ an "ideal" front-end processing matched to all the dominant multipaths and corresponding direction of arrivals (DOA's). In addition to suffering from high computational complexity in a dense multipath environment, such receivers rely heavily on accurate estimation of the delay and DOA parameters of dominant scatterers.

We introduce a canonical characterization of the received signal in terms of the essential degrees of freedom in the channel that are observable at the receiver. These degrees of freedom are captured by a fixed underlying basis corresponding to certain discrete multipath delays, Doppler shifts, and DOA's of the signaling waveform. In addition to eliminating the need for estimating arbitrary delays, Doppler shifts and DOA's of dominant scatterers, the canonical space-time receivers dictated by our signal model deliver optimal performance at substantially lower complexity compared to existing "idealized" receivers, especially in dense multipath environments.

Section II briefly derives the canonical channel characterization and Section III discusses some implications and illustrates its utility with a simple coherent receiver example.

Manuscript received October 26, 1998. The associate editor coordinating the review of this letter and approving it for publication was Prof. N. C. Beaulieu.

The authors are with the Department of Electrical and Computer Engineering, University of Wisconsin-Madison, Madison, WI 53706 USA (e-mail akbar@engr.wisc.edu; eko@cae.wisc.edu; vanveen@engr.wisc.edu).

Publisher Item Identifier S 1089-7798(99)04261-1.

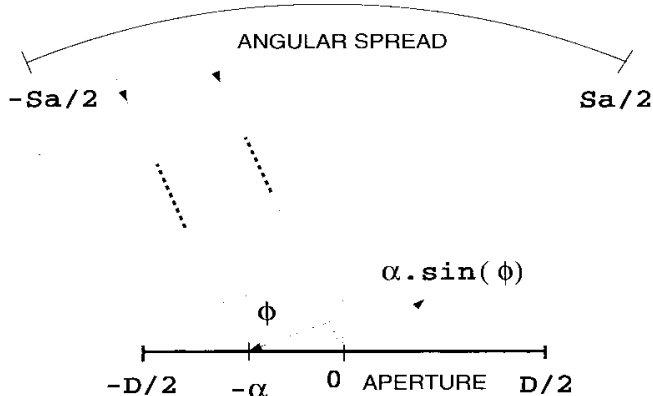

Fig. 1. Signal reception geometry.

\section{CANONICAL ChANNEL CHARACTERIZATION}

Consider a sensor array with aperture $D$. We develop the signal model using continuous aperture, as illustrated in Fig. 1. $S_{a}$ denotes the angular spread of the scatterers associated with the desired signal. For clarity of exposition, we characterize the channel effects on a single symbol. The complex baseband signal received at location $\alpha$ in the aperture is

$$
s_{\alpha}(t)=\int_{-S_{a} / 2}^{S_{a} / 2} e^{-j(2 \pi \sin (\phi) \alpha / \lambda)} x_{\phi}(t) d \phi, \quad-\frac{D}{2} \leq \alpha \leq \frac{D}{2}
$$

where $x_{\phi}(t)$ denotes the signal arriving from direction $\phi$ and $\lambda$ denotes the carrier wavelength. The signal $x_{\phi}(t)$ is related to the transmitted symbol waveform $q(t)$ via the angledependent time-varying channel impulse response $h_{\phi}(t, \tau)$ or, equivalently, the multipath-Doppler spreading function $H_{\phi}(\theta, \tau)$ [2], [3]

$$
\begin{aligned}
x_{\phi}(t) & =\int_{0}^{T_{m}} h_{\phi}(t, \tau) q(t-\tau) d \tau \\
& =\int_{0}^{T_{m}} \int_{-B_{d}}^{B_{d}} H_{\phi}(\theta, \tau) q(t-\tau) e^{j 2 \pi \theta t} d \theta d \tau
\end{aligned}
$$

where $T_{m}$ and $B_{d}$ denote the multipath and Doppler spreads, respectively. ${ }^{1}$

Due to the finite duration $T$ and essentially finite bandwidth $B$ of $q(t)$, the signal $x_{\phi}(t)$ exhibits only a finite number of temporal degrees of freedom that are captured by a set of uniformly spaced discrete multipath delays and Doppler shifts [4]. Similarly, the finite aperture dictates that $s_{\alpha}(t)$ possesses only a finite number of spatial degrees of freedom that are

\footnotetext{
${ }^{1}$ We note that $T_{m}$ and $B_{d}$ denote the maximum spreads-the variation of spreads with $\phi$ is captured by $H_{\phi}(\theta, \tau)$.
} 
captured by certain discrete DOA's. The following canonical space-time characterization of $s_{\alpha}(t)$ identifies the essential spatio-temporal degrees of freedom in the channel that are observable at the receiver.

\section{Canonical Channel Representation}

The signal $s_{\alpha}(t)$ admits the equivalent representation

$$
\begin{gathered}
s_{\alpha}(t) \approx \frac{1}{D T B} \sum_{l=0}^{L} \sum_{m=-M}^{M} \sum_{p=-P}^{P} \tilde{H}_{p / D}\left(\frac{m}{T}, \frac{l}{B}\right) q_{\alpha}^{m l p}(t), \\
0 \leq t \leq T ; \quad|\alpha| \leq D / 2
\end{gathered}
$$

in terms of the space-time basis waveforms

$$
q_{\alpha}^{m l p}(t)=q\left(t-\frac{l}{B}\right) e^{j(2 \pi m t / T)} e^{-j(2 \pi p \alpha / D)} .
$$

The coefficients $\tilde{H}_{p / D}(m / T, l / B)$ are uniformly spaced samples of the smoothed spreading function

$$
\begin{aligned}
\tilde{H}_{\zeta}(\theta, \tau)= & D T B \int_{-\tilde{S}_{a}}^{\tilde{S}_{a}} \int_{-B_{d}}^{B_{d}} \int_{0}^{T_{m}} \bar{H}_{\zeta^{\prime}}\left(\theta^{\prime}, \tau^{\prime}\right) e^{-j \pi\left(\theta-\theta^{\prime}\right) T} \\
& \cdot \operatorname{sinc}\left(\left(\zeta-\zeta^{\prime}\right) D\right) \operatorname{sinc}\left(\left(\theta-\theta^{\prime}\right) T\right) \\
& \cdot \operatorname{sinc}\left(\left(\tau-\tau^{\prime}\right) B\right) d \theta^{\prime} d \tau^{\prime} d \zeta^{\prime}
\end{aligned}
$$

where $\operatorname{sinc}(x)=\sin (\pi x) /(\pi x)$ and $\bar{H}_{\zeta}(\theta, \tau)=$ $\left(1 / \sqrt{\left.1 / \lambda^{2}-\zeta^{2}\right)} H_{\sin ^{-1}(\zeta \lambda)}(\theta, \tau)\right.$. The number of terms in (3) are determined by $L=\left\lceil T_{m} B\right\rceil, M=\left\lceil T B_{d}\right\rceil$ and $P=\left\lceil D \widetilde{S}_{a}\right\rceil$ where $\widetilde{S}_{a}=\sin \left(S_{a} / 2\right) / \lambda$.

Proof (sketch) [5]: Via a change of variables $\phi \rightarrow \zeta: \zeta=$ $\sin (\phi) / \lambda$ in $(1), s_{\alpha}(t)$ can be written as

$$
\begin{aligned}
s_{\alpha}(t)= & \int_{-\tilde{S}_{a}}^{\tilde{s}_{a}} \int_{-B_{d}}^{B_{d}} \int_{0}^{T_{m}} \bar{H}_{\zeta}(\theta, \tau) q(t-\tau) \\
& \cdot e^{j 2 \pi \theta t} e^{-j 2 \pi \zeta \alpha} d \zeta d \theta d \tau \\
= & \int g_{\alpha}(t, f) Q(f) e^{j 2 \pi f t} d f
\end{aligned}
$$

where $Q(f)$ is the Fourier transform of $q(t)$ and

$$
g_{\alpha}(t, f)=\iiint \bar{H}_{\zeta}(\theta, \tau) e^{-j 2 \pi \zeta \alpha} e^{j 2 \pi \theta t} e^{-j 2 \pi \tau f} d \zeta d \theta d \tau \text {. }
$$

In general, $g_{\alpha}(t, f)$ has infinite support in $t, f$, and $\alpha$. However, due to the finite array aperture $D$, and finite duration $T$ and approximately finite bandwidth $B$ of $q(t)$, only a corresponding "gated" version $\tilde{g}_{\alpha}(t, f)$ of $g_{\alpha}(t, f)$ matters in (6), which we express as the Fourier series

$$
\begin{gathered}
\tilde{g}_{\alpha}(t, f)=\sum_{l} \sum_{m} \sum_{p} c_{m l p} e^{j(2 \pi m t / T)} e^{-j(2 \pi l f / B)} e^{-j(2 \pi p \alpha / D)}, \\
0 \leq t \leq T ;|f| \leq B / 2 ;|\alpha| \leq D / 2 .
\end{gathered}
$$

It follows from (7) that the series coefficients are given by the samples of $\tilde{H}_{\zeta}(\theta, \tau)$ in (5)

$$
\begin{aligned}
c_{m l p}= & \int_{-D / 2}^{D / 2} \int_{0}^{T} \int_{-B / 2}^{B / 2} g_{\alpha}(t, f) e^{-j(2 \pi m t / T)} e^{j(2 \pi l f / B)} \\
& \cdot e^{j(2 \pi p \alpha / D)} d \alpha d t d f \\
= & \tilde{H}_{p / D}\left(\frac{m}{T}, \frac{l}{B}\right)
\end{aligned}
$$



Fig. 2. A schematic depicting the canonical space-time coordinates.

Substituting (8) and (9) in (6) yields (3). The constants $L=$ $\left\lceil T_{m} B\right\rceil, M=\left\lceil T B_{d}\right\rceil$ and $P=\left\lceil D \widetilde{S}_{a}\right\rceil$ correspond to the normalized multipath Doppler and angular spreads.

Fig. 2 illustrates the canonical space-time coordinates defined by the multipath-Doppler-angle sampling in the above representation. For a discrete $R$-dimensional sensor array, the canonical space-time basis functions (4) become

$$
\begin{array}{r}
\boldsymbol{q}_{m l p}(t)=\boldsymbol{a}\left(\phi_{p}\right) q\left(t-\frac{l}{B}\right) e^{j(2 \pi m t / T)}, \quad p=-P, \cdots, P ; \\
l=0, \cdots, L ; m=-M, \cdots, M
\end{array}
$$

where $\left\{\boldsymbol{a}\left(\phi_{p}\right)\right\}$ are the canonical array manifold vectors, which take the following form for a uniform linear array with spacing $d$

$\boldsymbol{a}(\phi)=\left[1, e^{-j 2 \pi(d \sin (\phi) / \lambda)}, \cdots, e^{-j 2 \pi(R-1)(d \sin (\phi) / \lambda)}\right]^{T} / \sqrt{R}$.

The angles $\left\{\phi_{p}\right\}$ corresponding to the canonical spatial sampling in (4) are governed by the relationship $\sin \left(\phi_{p}\right)=$ $(\lambda p / D)$. The corresponding canonical signal characterization for an $R$-sensor array is

$$
\boldsymbol{s}(t) \approx \sum_{l=0}^{L} \sum_{m=-M}^{M} \sum_{p=-P}^{P} H_{m l p} \boldsymbol{q}_{m l p}(t), 0 \leq t \leq T
$$

for some canonical channel coefficients $H_{m l p}$.

\section{DISCUSSION}

While the canonical representation (12) is quite general, it is particularly advantageous in the context of spread spectrum $(T B \gg 1)$ signaling [5], [6]. First, it provides a robust and maximally parsimonious characterization of space-time propagation effects in terms of the fixed basis (4). It eliminates the need for estimating arbitrary delays, Doppler shifts and DOA's of dominant scatterers. ${ }^{2}$ Second, the representation provides a versatile framework for channel modeling - both deterministic and stochastic. In particular, the $(L+1)(2 M+$ 1) $(2 P+1)$ dimensional canonical coordinates defined by the basis (10) characterize the inherent diversity afforded by a wide-sense stationary uncorrelated scatterer (WSSUS) channel [5], [4]. ${ }^{3}$ Finally, the representation (12) induces a

\footnotetext{
${ }^{2}$ Up to synchronization to a "global" delay, Doppler offset and DOA to "align" the basis, which is required in all receivers.

${ }^{3}$ The special case of canonical multipath-Doppler coordinates in time-only processing is discussed in [4] and [6]
} 
canonical subspace structure that can be fruitfully exploited for interference suppression in multiuser scenarios [5], [6].

The main source of error in (3) is due to the bandlimited approximation, which can be made arbitrarily small by sufficient oversampling [5]. For a direct sequence CDMA system, $B$ is inversely proportional to the chip duration $T_{c}$, and in the following we use $B=\mathcal{O} / T_{c}$ where $\mathcal{O}$ is the oversampling factor, typically 2,4 , or 8 . The choice $\mathcal{O}=1$ can generate an approximately orthonormal basis, albeit at the expense of a loss of accuracy in (12) [5], [7]. The accuracy of (12) can be improved by increasing $\mathcal{O}$, although at the expense of losing orthogonality [4], [5].

We illustrate the advantage of the canonical space-time coordinates with a simple example of single-user coherent BPSK signaling over a slow fading channel (negligible Doppler effects). An $R=9$ element uniform linear array is assumed with half-wavelength spacing. The $R$-dimensional complex baseband signal for one symbol is given by $\boldsymbol{r}(t)=\boldsymbol{s}(t) \boldsymbol{b}+\boldsymbol{n}(t)$, $0 \leq t \leq T$, where $b$ is the data symbol and $\boldsymbol{n}(t)$ denotes a zeromean complex white Gaussian noise process. The signal $\boldsymbol{s}(t)$ is modeled as $\Sigma_{l=1}^{L_{T}} \beta_{l} a\left(\phi_{l}\right) q\left(t-\tau_{l}\right)$ where $\phi_{l}$ and $\tau_{l}$ are the DOA and delay corresponding to the $l$ th path. $L_{T}$ denotes the total number of multipaths, and the fading coefficients $\left\{\beta_{l}\right\}$ are uncorrelated.

Conventional coherent space-time receivers, such as those proposed in [1], are based on the "ideal" test statistic

$$
Z=\operatorname{sign}\left(\operatorname{Re}\left\{\sum_{l=1}^{L_{T}} \hat{\beta}_{l}^{*} \int \boldsymbol{a}^{H}\left(\hat{\phi}_{l}\right) \boldsymbol{r}(t) q^{*}\left(t-\hat{\tau}_{l}\right) d t\right\}\right)
$$

which performs matched-filtering to all the multipath components and requires estimates of $\phi_{l}, \tau_{l}$, and $\beta_{l}$ for each multipath. The canonical representation (12) provides an new equivalent characterization of the ideal statistic (13) that eliminates the need for DOA and delay estimates

$$
Z=\operatorname{sign}\left(\operatorname{Re}\left\{\sum_{l=0}^{L} \sum_{p=-P}^{P} \hat{H}_{0 l p}^{*} \int \boldsymbol{q}_{\mathrm{Olp}}^{H}(t) \boldsymbol{r}(t) d t\right\}\right)
$$

Note that the canonical receiver only requires estimates of the fading coefficients $\left\{H_{0 l p}\right\}$, and the number of canonical coordinates, $(L+1)(2 P+1)$, can be substantially smaller than the number of physical coordinates, $L_{T}$, especially in dense multipath environments. For comparison purposes, we assume perfect parameter estimates in both receivers. The coefficients $\left\{\hat{H}_{0 l p}\right\}$ are obtained by projecting the noise-free signal $\boldsymbol{s}(t)$ onto the canonical subspace [5].

A length-31 $M$ sequence is used as the spreading code in the following numerical example. A dense multipath environment is simulated using a total of $21 \times 16$ scatterers distributed evenly over $(\phi, \tau) \in$ $[-\pi / 10, \pi / 10] \times\left[0,0.9375 T_{c}\right]$. The canonical representation samples at DOA's $\sin ^{-1}([-4 / 9,-2 / 9,0,2 / 9,4 / 9])=$ $\{-0.461,-0.224,0,0.224,0.461\}$ radians, and delays $\left\{0,\left(T_{c} / \mathcal{O}\right),\left(2 T_{c} / \mathcal{O}\right), \cdots, T_{c}\right\}$ with $\mathcal{O}=1,2,4,8$. Fig. 3 compares the performance of the conventional (ideal) and canonical receivers. At a symbol error probability $10^{-4}$, the

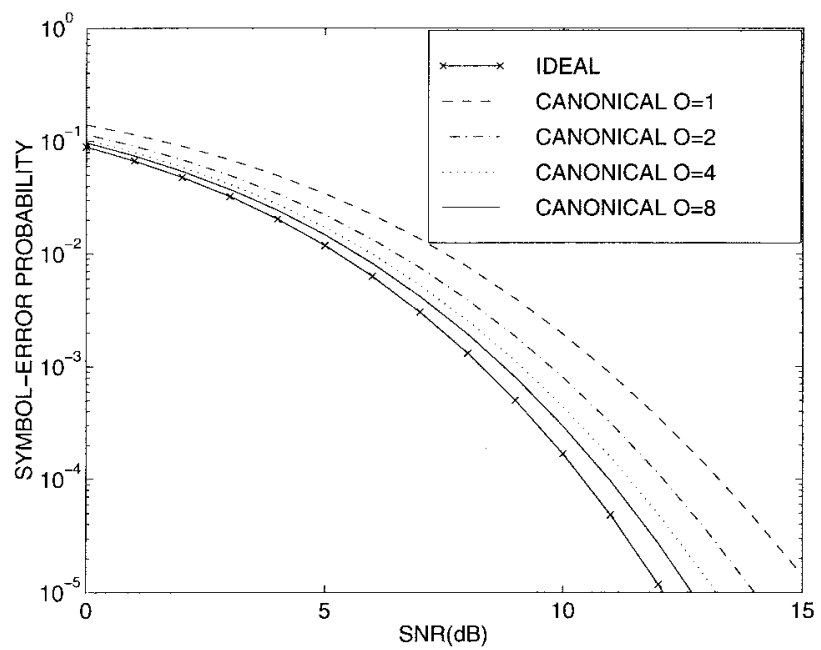

Fig. 3. Space-time processing: conventional (ideal) versus canonical for different $\mathcal{O}$ s.

canonical receiver is within $1 \mathrm{~dB}$ of the ideal receiver for $\mathcal{O}=4$ or 8 , and this gap decreases with increasing $\mathcal{O}$ [5]. Note that the canonical receiver delivers this near-optimal performance at a substantially reduced complexity. The ideal receiver requires $21 \times 16 \times 3$ estimates of $\left(\phi_{l}, \tau_{l}, \beta_{l}\right)$, and computation of $21 \times 16$ matched space-time filter outputs. In contrast, the canonical receiver for $\mathcal{O}=8$ only requires estimates of $4 \times 9$ coefficients $\hat{H}_{0 l p}$ and computation of $4 \times 9$ matched filter outputs. Furthermore, the canonical matched filter outputs can be efficiently computed via a space-time RAKE receiver structure [5].

All practical receivers have limited operational bandwidth which limits the accuracy of closely spaced delay estimates in a dense multipath environment and also limits the benefits of oversampling. Even if joint angle-delay estimation frameworks [1] are employed, a large number of observations and relatively complex algorithms are necessary to obtain accurate parameter estimates for the "idealized" conventional receivers. The canonical channel characterization introduced here dictates receivers that have dramatically reduced complexity and are likely to be far more robust to channel estimation errors associated with limited data and the presence of noise.

\section{REFERENCES}

[1] A. J. Paulraj and C. B. Papadias, "Space-time processing for wireless communications," IEEE Signal Processing Mag., pp. 49-83, Nov. 1997.

[2] J. G. Proakis, Digitial Communications, 3rd ed. New York: McGraw Hill, 1995.

[3] P. A. Bello, "Characterization of randomly time-variant linear channels," IEEE Trans. Commun. Syst., vol. COM-11, pp. 360-393, 1963.

[4] A. M. Sayeed and B. Aazhang, "Joint multipath-Doppler diversity in mobile wireless communications," IEEE Trans. Commun., vol. 47, pp. 123-132, Jan. 1999

[5] E. N. Onggosanusi, A. M. Sayeed, and B. D. Van Veen, "Canonical space-time processing in wireless communications," IEEE Trans. Commun., vol. 47, Apr. 1999.

[6] A. M. Sayeed, "Canonical multipath-Doppler coordinates in wireless communications," in Proc. 36th Annu. Allerton Conf. on Communication, Control and Computing, 1998, pp. 536-545.

[7] _ "Canonical space-time processing in CDMA systems," in Proc. 1999 IEEE Int. Conf. on Acoust., Speech, and Signal Processing-ICASSP '99, vol. 5, pp. 2611-2614. 